\title{
CONFLICT OF INTEREST COMPLIANCE ARTICLE 5
}

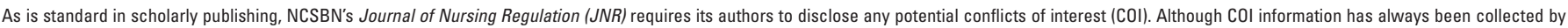
our staff in order to support editors' review of the paper, it was not our standard practice to publish COI statements in each article. In this issue, JNR is retrospectively publishing the COI statements, which were collected with the below papers at submission, in order to make potential COl's transparent to readers, as well as editors.

1. “Outcomes of Substance Use Disorder Monitoring Programs for Nurses" [Journal of Nursing Regulation, 2020;11(2):28-35] https://doi. org/10.1016/S2155-8256(20)30107-1

org/10.1016/S2155-8256(20)30107-1 Declaration of competing interest: The authors declare that they have no known competing financial interests or personal relationships that could have appeared to influence the work reported in this paper.

2. "Trends and Challenges in Regulating Nursing Practice: 10 Years Later" [Journal of Nursing Regulation, 2020;11(1):12-20] https://doi. org/10.1016/S2155-8256(20)30055-7

Declaration of competing interest: The authors declare that they have no known competing financial interests or personal relationships that could have appeared to influence the work reported in this paper.

3. "Outcomes and Impact of a Nursing Regulatory Orientation Workshop for Nurse Leaders" [Journal of Nursing Regulation, 2020;10(4):22-29] https://doi.org/10.1016/S2155-8256(20)30010-7 Declaration of competing interest: The authors declare that they have no known competing financial interests or personal relo ships that could have appeared to influence the work reported in this paper.

4. "Nurse Faculty Workload Characteristics: A State-Level Survey" [Journal of Nursing Regulation, 2020;11(2):12-19] https://doi. [Journal of Nursing Regulation,
org/10.1016/S2155-8256(20)30105-8

org/10.1016/S2155-8256(20)30105-8
Declaration of competing interest: The authors declare that they Declaration of competing interest: The authors declare that they
have no known competing financial interests or personal relationhave no known competing financial interests or personal relation-
ships that could have appeared to influence the work reported in this ships thatc
paper.

5. "Ensuring Effective and Quality Care During a Pandemic" [Journal of Nursing Regulation, 2020;11(1):58-60] https://doi.org/10.1016/ S2155-8256(20)30062-4

Declaration of competing interest: The authors declare that they have no known competing financial interests or personal relationships that could have appeared to influence the work reported in this paper.
6. "Strategies for and Barriers to Fatigue Management Among Acute Care Nurses" [Journal of Nursing Regulation, 2020;11(2):36-43] Care Nurses" [Journal of Nursing Regulation,
https://doi.org/10.1016/S2155-8256(20)30108-3

https://doi.org/10.1016/S2155-8256(20)30108-3 Declaration of competing interest: The authors declare that they
have no known competing financial interests or personal relationhave no known competing financial interests or personal relation-
ships that could have appeared to influence the work reported in this phips ther.

7. “NCSBN Regulatory Guidelines and Evidence-Based Quality Indicators for Nursing Education Programs" [Journal of Nursing Regulation, 2020;11(2S):S1-S64] https://doi.org/10.1016/ S2155-8256(20)30075-2

Declaration of competing interest: The authors declare that the have no known competing financial interests or personal relationships that could have appeared to influence the work reported in this paper.

8. "Medication Disposal Standards of Care in a Correctional Facility" [Journal of Nursing Regulation, 2020;11(1):21-23] https://doi. org/10.1016/S2155-8256(20)30056-9

Declaration of competing interest: The authors declare that they have no known competing financial interests or personat relationships that could have appeared to influence the work reported in this paper.

9. "Early Career Nurse Reports of Work-Related Substance Use" [Journal of Nursing Regulation, 2020;11(1):29-35] https://doi.org/10.1016/ nal of Nursing Regulation,

Declaration of competing interest: The authors declare that they Declaration of competing interest: The authors declare that they
have no known competing financial interests or personal relationhave no known competing financial interests or personal relation-
ships that could have appeared to influence the work reported in this ships that
paper.

10. “Components of Nurse Substance Use Disorder Monitoring Programs" [Journal of Nursing Regulation, 2020;11(2):20-27] https://doi org/10.1016/S2155-8256(20)30106-X

Declaration of competing interest: The authors declare that they have no known competing financial interests or personal relationships that could have appeared to influence the work reported in this paper.
11. “Opioid Prescribing Safety Measures Utilized by Primary Healthcare Providers in Canada: A Scoping Review" "[Journal of Nursing Regu Providers in Canada. A Scoping Review " Journal of Nursing Regulation, 2020;10(4):13-21] https://doi.org/10.1016/S2155-8256(20)30009-0 Declaration of competing interest: The authors declare that they have no known competing financialinterests or personal relationships that could have appeared to influence the work reported in this
paper.

12. “Cybersecurity: Nurses on the Front Line of Prevention and Education" [Journal of Nursing Regulation, 2020;10(4):48-53] https://doi. org/10.1016/S2155-8256(20)30014-4

Declaration of competing interest: The authors declare that they have no known competing financial interests or personal relationships that could have appeared to influence the work reported in this paper.

13. “Nurse Practitioner Student Perceptions of Face-to-Face and Telehealth Standardized Patient Simulations" [Journal of Nursing Regulation, 2020;10(4):37-44] https://doi.org/10.1016/ S2155-8256(20)30012-0

Declaration of competing interest: The authors declare that they have no known competing financial interests or personal relationships that could have appeared to influence the work reported in this paper.

14. "Access to Care in New Jersey: Making the Case for Modernizing Legislation" [Journal of Nursing Regulation, 2020;11(1):36-41] https:// doi.org/10.1016/S2155-8256(20)30059-4

doi.org/10.1016/S2155-8256(20)30059-4 have no known competing financial interests or personal relationships that could have appeared to influence the work reported in this paper.

15. "Task Shifting: A High-Level Analysis of Scholarship" [Journal of Nursing Regulation, 2020;11(2):4-11] https://doi.org/10.1016/ S2155-8256(20)30104-6

Declaration of competing interest: The authors declare that they have no known competing financial interests or personal relationships that could have appeared to influence the work reported in this paper. 